\title{
The Zugzwang Hypothesis: Why Human Lifespan Cannot Be Increased
}

\author{
Syed Ibrahim Rizvi \\ Department of Biochemistry, Faculty of Science, University of Allahabad, Allahabad, India
}

\section{Keywords \\ Human · Lifespan · Zugzwang $\cdot$ Longevity}

\section{Introduction}

Lifespan is one of the most variable life history traits in the animal kingdom, lasting from days to centuries. Despite intensive investigation, there are still many grey areas in our understanding of the factors which contribute to the variability of lifespan $[1,2]$. Humans are among the fortunate animals which have an unusually long lifespan compared to their similar sized mammals. On the flip side, the long lifespan of humans and large genetic heterogeneity are important reasons why it is very difficult to use humans as models to study ageing or longevity or test the efficacy of anti-ageing interventions. Ageing studies on humans often require a very large cohort of people and can potentially be affected by many confounding factors [3]. As a consequence, most studies involving ageing, lifespan, and anti-ageing interventions are based on model systems $[4,5]$.

In the evolutionary history after divergence from the great apes, the most recent of our primate ancestors, humans have completed almost 300,000 generations. Dur-

karger@karger.com

(C) 2021 S. Karger AG, Basel

www.karger.com/ger

Karger! ing this period, the lifespan of $H$. sapiens has almost doubled [6]. The increased longevity of humans is, in part, attributable to environmental changes; improved food, water, and hygiene; reduced impact of infectious disease; and improved medical care at all ages. However, the above factors had an opportunity to play some role in increasing lifespan only in the last 2 centuries. The dramatic increase in human lifespan compared to our nearest ancestors, should, therefore, must have other valid explanations. It is highly conceivable that forces of natural selection may have played vital role in increasing the basic longevity of humans.

The evolution of humans as a distinct species, a conservative estimate of approximately 200,000 years, has provided a decent time period to the forces of natural selection to work on extending human lifespan much beyond the constraints of the laws which govern lifespan in other animals. It is universally acknowledged that the force of natural selection explains the diversity of organisms on this earth. However, the evolutionary progress of humans is sometimes beyond the paradigm of natural selection. Although all higher organisms display commonality in basic physiology, biochemistry, and, to an extent, anatomy, no other animal, except $H$. sapiens, has tried to find ways to extend its lifespan. 
The most dramatic deviation of humans from animals has been the evolution of mind and the resulting consequences. While for other organisms, natural selection always played on basic needs selecting and propelling evolution into forward direction on expected lines, the increased intellect of $H$. sapiens provided many new targets for natural selection to work.

Two prominent developments in the evolutionary history of humans provided natural selection to devise mechanisms to extend human lifespan much beyond similar-sized animals. The evolution of language and the resultant assimilation and transfer of knowledge between two generations was a far greater beneficial trait to be conserved. There would have been a trade-off between the ageing of the organism and increased longevity even at the cost of loss of physical prowess. It is thus plausible that a longer lifespan extending much beyond the reproductive period provided more time for propagation of knowledge. The benefits of this longer lifespan thus created a situation in which evolutionary forces through the process of natural selection acted to conserve a longer lifespan. The extension of lifespan was nevertheless governed by the inherent ageing process, and therefore, a limit was to be defined. As a result, while human lifespan increased with respect to similar-sized animals to achieve a specific purpose, it achieved a limiting value after which it was too costly to maintain soma.

Although studies in model organisms have shown that maximum lifespan can be flexible and may be affected by genetic and pharmacological interventions [7], an analysis of global demographic data has revealed that the maximum lifespan of humans has not increased since 1990s [8]. It was Olshansky et al. [9] who estimated the upper limits to human longevity based on hypothetical reductions in mortality rates and hypothesized that that life expectancy at birth would not exceed 85 years. This has led to a conclusion that the maximum lifespan of humans is fixed and subject to natural constraints [8]. For humans, the maximum reported age at death is accepted to be set at 122 years, the age at death of Jeanne Calment. However, this figure is also being debated in view of recent controversy regarding the original identity of Jeanne Calment [10].

Extending an organism's lifespan, through selection of longer living individuals, required tinkering with all pathways and signalling events which control longevity and were constitutively conserved in eukaryotes. As time progressed and eukaryotes became more complicated systems, there arose the need for signalling pathways that could monitor the function and regulate cell growth and proliferation. Two major evolutionary developments happened which played vital roles in the organism's ability to regulate cell growth in response to the availability of energy and nutrients. The AMP-activated protein kinase and target of rapamycin became the focal point in regulating energy production, sensing availability of nutrients, and regulating growth. These signalling pathways thus became the primary internal regulators of cell growth and proliferation.

Intensive research over the years has now resulted in the identification several major pathways and signalling events which play decisive roles in determining lifespan. The putative targets identified to control lifespan include AMP-activated protein kinase, target of rapamycin, sirtuin, insulin/insulin-like growth factor signalling, and a host of transcription factors $[11,12]$. All major interventions aimed to achieve anti-ageing effects have been based on modulating the above-mentioned signals/proteins.

It is interesting to understand that all intervention strategies, where experimental success has been shown, are limited to alleviating some age-dependent disease. Targeting Alzheimer's, Parkinson's, dementia, cardiovascular disease, cancer, hypertension, osteoporosis, or any other old age ailment is a piecemeal approach and cannot be classified as life extension. Dietary polyphenols have been one of the most misfired therapies in the existing approaches towards finding something which can extend lifespan. While polyphenols have been extensively studied on model organisms and a host of beneficial effects attributed to their consumption, there is still no direct evidence of dietary polyphenols causing anti-ageing effects on humans [13].

\section{Pertinent Questions}

What could be the biological causes of limit to human lifespan? Why anti-ageing interventions that work on lower organisms may not work on humans? To answer these questions, I propose the following hypothesis.

\section{The Zugzwang Hypothesis}

Zugzwang is a German word with the literal meaning "compulsion to move." This word is frequently used in chess to describe a situation when a player gets a disadvantage because it is his turn to play, but all the available moves are bad. In Zugzwang position, any move the player makes will clearly weaken his position. Here, I propose 
that at this stage of evolution, humans may face the Zugzwang problem. Scientific research and the understanding of the hallmarks of ageing [14] now provide humans with more than a dream to extend lifespan. However, it must be taken into consideration that natural selection has already played its part in extending human lifespan much beyond the expectation. All possible mechanisms which can increase longevity in lower animals have already been exploited by natural selection to stretch human lifespan. Any artificial attempt to tinker, through any possible intervention, with the signalling pathways or transcription factors to achieve a longer lifespan may actually be disadvantageous to humans. H. sapiens may thus be considered to be in the Zugzwang state. Humans may have already achieved or approached the maximum lifespan, and further lifespan extension may be very difficult or impossible. Documented record of human longevity for the last 100 years (with a conservative estimate of data from 8 billion individuals) shows that the limit of human lifespan is around 122 years; the fact that no individual has lived beyond this limit is a clue to the validity of the Zugzwang hypothesis.

\section{Statement of Ethics}

No experimental work performed on animals or humans. No ethical compliance or approval required.

\section{Conflict of Interest Statement}

There are no conflicts of interest.

\section{Funding Sources}

This work was supported by a research grant to SIR from SERBDST, government of India (EMR/2016/006470). The Department of Biochemistry, University of Allahabad, is supported by grants from the Department of Science and Technology (FIST) and University Grants Commission (SAP), government of India.

\section{Author Contributions}

S.I. Rizvi conceived the idea and wrote the manuscript.

\section{References}

1 Johnson AA, Shokhirev MN, Shoshitaishvili B. Revamping the evolutionary theories of aging. Ageing Res Rev. 2019;55:100947.

2 Kishimoto S, Uno M, Nishida E. Molecular mechanisms regulating lifespan and environmental stress responses. Inflamm Regen. 2018;38:22.

3 Taormina G, Ferrante F, Vieni S, Grassi N, Russo A, Mirisola MG. Longevity: lesson from model organisms. Genes. 2019;10(7): 518.

4 Petralia RS, Mattson MP, Yao PJ. Aging and longevity in the simplest animals and the quest for immortality. Ageing Res Rev. 2014; 16:66-82.

5 Kumar Chaudhary M, Rizvi SI. Invertebrate and vertebrate models in aging research. Biomed Pap. 2019;163(2):114-21.
6 Finch CE. Evolution in health and medicine Sackler colloquium: evolution of the human lifespan and diseases of aging: roles of infection, inflammation, and nutrition. Proc Natl Acad Sci U S A. 2010;107(Suppl 1):1718-24.

7 Kenyon C. The plasticity of aging: insights from long-lived mutants. Cell. 2005;120(4): 449-60.

8 Dong X, Milholland B, Vijg J. Evidence for a limit to human lifespan. Nature. 2016; 538(7624):257-9.

9 Olshansky SJ, Carnes BA, Cassel C. In search of Methuselah: estimating the upper limits to human longevity. Science. 1990;250(4981): 634-40.
10 Zak N, Gibbs P. A bayesian assessment of the longevity of Jeanne Calment. Rejuvenation Res. 2020;23(1):3-16.

11 Martínez Corrales G, Alic N. Evolutionary conservation of transcription factors affecting longevity. Trends Genet. 2020;36(5):373-82.

12 Pan H, Finkel T. Key proteins and pathways that regulate lifespan. J Biol Chem. 2017; 292(16):6452-60.

13 Pallauf K, Duckstein N, Rimbach G. A literature review of flavonoids and lifespan in model organisms. Proc Nutr Soc. 2017;76(2):14562.

14 López-Otín C, Blasco MA, Partridge L, Serrano $M$, Kroemer $G$. The hallmarks of aging. Cell. 2013;153(6):1194-217. 